Klaus J. Zink

\section{Zum Selbstverständnis der Gesellschaft für Arbeitswissenschaft e.V. (GfA)}

Veränderte Rahmenbedingungen stellen neue Anforderungen - auch an die Arbeitswissenschaft. Konsequenterweise muss sich auch die Gesellschaft für Arbeitswissenschaft mit der Frage auseinander setzen, wofür sie steht und welches ihre Objektbereiche und Zielgruppen sind. Dies beinhaltet auch die Auseinandersetzung mit normativen Fragestellungen, die - wie in anderen wissenschaftlichen Gesellschaften meist nur implizit vorhanden war.

Das Ergebnis eines längeren, partizipativ geführten Diskussionsprozesses ist im Folgenden wiedergegeben. ${ }^{1}$

Die Gesellschaft für Arbeitswissenschaft e.V. will durch die Förderung disziplinübergreifender, ganzheitlicher Forschungs- und Gestaltungskonzepte einen relevanten Beitrag zur Gestaltung der Qualität der Lebens- und Arbeitsbedingungen leisten.

\section{Umsetzung des Leitbildes}

\section{Ganzheitliche, disziplinübergreifende Konzepte}

Die Analyse, Beurteilung und Gestaltung menschlicher Arbeit, aber auch die Gestaltung menschengerechter Produkte, Dienstleistungen, Systeme und Umwelten erfordern das Wissen aus verschiedenen wissenschaftlichen und praxisorientierten Einzeldisziplinen.

Dazu zählen u.a. Arbeits- und Organisationspsychologie, Arbeitsmedizin, Arbeitsphysiologie, Arbeitssoziologie, Arbeitspolitik, Arbeitspädagogik sowie Ingenieur-, Wirtschafts- und Rechtswissenschaften.

Die Gesellschaft für Arbeitswissenschaft e.V. führt dieses Wissen mit wissenschaftlichem Anspruch zusammen. Das heißt, ihre Besonderheit bezieht die Arbeitswissenschaft - und damit auch die GfA - aus der Zusammenführung der Einzeldisziplinen (Arbeitswissenschaften) zu einer ganzheitlichen Sicht.
Ganzheitlich bedeutet in diesem Zusammenhang nicht nur die Integration von Einzeldisziplinen, sondern auch die Vereinbarkeit unterschiedlicher Zielsetzungen, wobei humane und wirtschaftliche Ziele eine besondere Rolle spielen.

Human ist eine Arbeit dann, wenn sie menschengerecht und menschenwürdig ausgeführt werden kann, und damit die physische und psychische Gesundheit nicht beeinträchtigt und auch das Wohlbefinden nicht nachhaltig stört. Sie sollte den Bedürfnissen und Qualifikationen entsprechen und einen individuellen und/oder kollektiven Einfluss auf die Arbeit ermöglichen. Schließlich ist eine Entwicklung der Persönlichkeit durch Arbeit im Sinne einer Entfaltung von Potentialen und Förderung von Kompetenzen anzustreben.

Bei der Beurteilung der Wirtschaftlichkeit muss berücksichtigt werden, dass Lösungswege auf der Ebene einer einzelnen Organisation ,wirtschaftlich“ sein können, sich aber auf der volkswirtschaftlichen oder gesellschaftlichen Ebene als „,unwirtschaftlich“ erweisen können. Alle diese Beurteilungsebenen sollten deshalb berücksichtigt werden.

Die spezifische Aufgabe der GfA besteht in der Förderung der wissenschaftlichen und fachlichen Belange der Arbeitswissenschaft. Dazu pflegt sie die Verbindung unter den an der Arbeitwissenschaft Interessierten im deutschen Sprachraum und auf internationaler Ebene. Sie versteht sich dabei auch in besonderem Maße als aktives Forum für den Dialog zwischen Wissenschaft und Praxis sowie allen interessierten gesellschaftlichen Gruppen.

Die Gesellschaft für Arbeitswissenschaft e.V. entwickelt und sichert professionelle Standards (z.B. durch Mitwirkung in der Normung oder als deutscher Partner für die Zertifizierung zum Euro-Ergonom aber auch durch Begutachtungsverfahren für Zeitschriften- und Kongressbeiträge). So weit möglich und erforderlich vertritt sie die Interessen ihrer Mitglieder in fachlicher Hinsicht.

\section{Grundlagen der Umsetzung}

\section{Mensch und Arbeit in komplexen Systemen}

- Die (Arbeits-)Gesellschaft unterliegt einem ständigen Wandel, der eine entsprechende Weiterentwicklung der Arbeitswissenschaft erfordert. Da-

1 Die "Denkschrift" zum "Selbstverständnis der Gesellschaft für Arbeitswissenschaft e.V." kann bei der Geschäftsstelle der GfA, Ardeystraße 67, 44139 Dortmund, angefordert werden. 
bei werden Zielkonflikte und Spannungen auftreten und als normal akzeptiert.

- Arbeitswissenschaftliche Forschung und Gestaltung ist sich stets der Besonderheit des Menschen bewusst.

- Arbeitswissenschaftliches Handeln zielt auf eine vorausschauende an humanen und wirtschaftlichen Kriterien orientierte Gestaltung von Arbeit, Technik und Organisation.

- Arbeitswissenschaftliche Problemlösungen sind einem Konzept verpflichtet, das allen Akteuren (z.B. Beschäftigte, Management, Kapitalgeber, Gesellschaft) einen Nutzen bringt.

- Die von der Analyse, Beurteilung und Gestaltung betroffenen Personen werden - wo immer möglich - an diesen Prozessen beteiligt.

- Die Arbeitswissenschaft befasst sich auch mit der Analyse, Beurteilung und Gestaltung von NichtErwerbsarbeit (z.B. Hausarbeit, Eigenarbeit, Bürgerarbeit etc.).

- Die Gesellschaft für Arbeitswissenschaft e.V. bzw. deren Mitglieder fühlen sich dem gesellschaftlichen Ziel verpflichtet, wo immer möglich, Beiträge zur Erhaltung, Schaffung und (angemessenen) Verteilung von humaner, wirtschaftlicher und umweltverträglicher Arbeit $\mathrm{zu}$ leisten.

\section{Zielgruppen}

\section{Breites Spektrum}

Zielgruppe der GfA sind alle ,arbeitswissenschaftlich tätigen oder interessierten Akteure“. Diese nehmen einerseits in der Praxis Aufgaben der Analyse, Beurteilung und Gestaltung von Arbeit wahr, können andererseits aber auch an der Gestaltung von Produkten und Prozessen beteiligt sein. Schließlich sind alle, die Umsetzungsverantwortung tragen (z.B. Führungskräfte), hier einzubeziehen.

Arbeitswissenschaftliche Erkenntnisse werden an Universitäten bzw. Hochschulen und anwendungsorientierten Forschungsinstituten erarbeitet und verbreitet sowie durch die in Unternehmen tätigen Arbeitswissenschaftlerinnen und Arbeitswissenschaftler ergänzt und umgesetzt.

Auch Mitarbeiterinnen und Mitarbeiter in Verbänden, staatlichen Institutionen und Selbsthilfeorganisationen sind arbeitswissenschaftlich tätig. Die Zielgruppe der GfA ist dementsprechend weit gefasst. $\mathrm{Zu}$ den inhaltlichen Kernbereichen zählen:
- Arbeits- und Gesundheitsschutz

- Produktgestaltung

- Arbeits- und Organisationsgestaltung

- Organisationsentwicklung und Arbeitspolitik Geht man davon aus, dass jeder Mensch im Laufe seines Lebens mit vielfältigen Formen von Arbeit konfrontiert wird und sich täglich mit Ergebnissen von Arbeit (im Sinne von Produkten und Dienstleistungen) auseinanderzusetzen hat, dann wird das weite Spektrum arbeitswissenschaftlicher Themen deutlich.

Die GfA will dazu beitragen, diese Inhalte nicht nur unter ihren Mitgliedern zu verbreiten, sondern für möglichst viele Menschen zu erschließen.

Dazu ist es erforderlich, arbeitswissenschaftliche Erkenntnisse in die Ausbildung all jener zu integrieren, die diese Inhalte unmittelbar (z.B. in der Produktions- und Produktgestaltung, Arbeitsorganisation und betriebliche Organisationsentwicklung, im Arbeitsschutz, der Arbeitsgestaltung, der Personalentwicklung und in arbeitspolitisch bedeutsamen Bereichen) oder mittelbar (z.B. in der Ausbildung, Investitionsplanung, Einkauf, Personalabteilungen oder bei Führungsaufgaben) benötigen. Darüber hinaus sollten arbeitswissenschaftliche Erkenntnisse und Anliegen auch in allgemeinbildenden insbesondere aber in berufsbildenden Schulen vermittelt werden.

Die GfA setzt sich für den Transfer arbeitswissenschaftlichen Wissens durch die Organisation geeigneter „Plattformen“ (z.B. Kongresse, Workshops, Publikationen, Clearingstellen z.B. für Beratung, Stellenbörsen etc.) ein.

Prof. Dr. Klaus J. Zink

Lehrstuhl für Industriebetriebslehre und Arbeitswissenschaft

Past President der GfA

Universität Kaiserslautern

Gottlieb-Daimer-Straße

67663 Kaiserslautern 